\title{
Frühe Gabe senkt bei Prädisposition Typ-1-Diabetes-Risiko
}

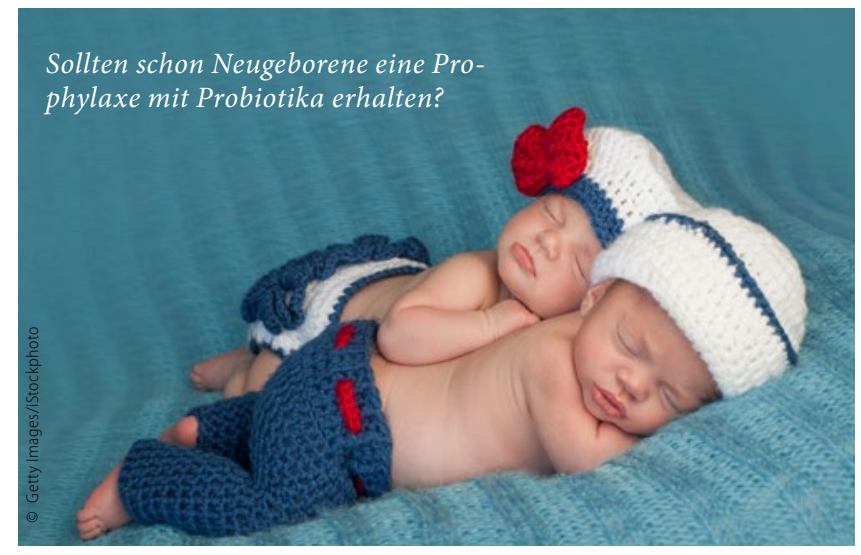

Fragestellung: Welche Assoziationen gibt es zwischen supplementär gegebenen Probiotika im ersten Lebensjahr und Inselautoimmunität bei Kindern mit erhöhtem genetischem Risiko für Typ-1-Diabetes?

Hintergrund: Es wird vermutet, dass Probiotika Immunantworten auf Umwelteinflüsse beeinflussen können, indem sie eine gesunde Mikrobiota unterstützen. Deshalb könnten sie theoretisch verwendet werden, um die Entwicklung einer mit Typ1-Diabetes (T1DM) assoziierten Inselautoimmunität zu verhindern.

\section{Originalie}

Uusitalo U, Liu X, Yang J et al. TEDDY Study Group. Association of Early Exposure of Probiotics and Islet Autoimmunity in the TEDDY Study. JAMA Pediatr. 2016 Jan 1;170(1):20-8.
Patienten und Methoden: Diese derzeit laufende prospektive Studie begann am 1. September 2004 und untersucht Kinder aus sechs klinischen Zentren von denen drei in den USA (Colorado, Georgia/ Florida, Washington) und drei in Europa
(Finnland, Deutschland, Schweden) liegen. Die Kinder werden longitudinal auf mit T1DM assoziierte Autoantikörper untersucht. Blutproben werden alle 3 Monate entnommen im Alter von 3-48 Monaten und danach alle 6 Monate gesammelt, um sie auf (anhaltende) Inselautoimmunität zu untersuchen. Einzelheiten der Nahrungszufuhr von Kleinkindern inklusive probiotische Supplemente und Beikost werden von der Geburt an untersucht mittels Fragebögen und Tagebüchern. In Form einer „Time-to-Event"-Analyse wurde die Assoziation von Probiotikagebrauch und Inselautoimmunität untersucht, wobei stratifiziert wurde nach Land und adjustiert auf positive Familienanamnese für T1DM, HLA-DR-DQ-Genotypen, Geschlecht, Geburtenfolge, Art der Entbindung, exklusives Stillen, Geburtsjahr, Antibiotikamedikation des Kindes, Diarrhoen, mütterliches Alter, Probiotikagebrauch und Rauchen. Insgesamt wurden 8676 Kinder unter 4 Monaten Lebensalter mit geeigneten Genotypen in die longitudinale Studie aufgenommen. 7473 Kinder in einem Alter von 4 bis 10 Jahren standen am Ende (31. Oktober 2014) zur Verfügung. Als Einflussfaktor wurde die frühe Einnahme von Probiotika untersucht. Der Endpunkt war Inselautoimmunität, die mittels spezifischen Inselautoantikörpern untersucht wurde.

Ergebnisse: Die frühe Gabe von Probiotika (im Lebensalter von 0-27 Tagen) war mit einem verminderten Risiko für Inselautoimmunität assoziiert, verglichen wurde dabei mit Probiotikagabe später als 27 Tagen oder fehlender Probiotikagabe. (Hazard Ratio [HR] 0,66; 95\%-KI, 0,46-0,94). Diese Assoziation trat bei den Kindern mit dem DR3/4 Genotypen auf (HR 0,40; 95\%-KI $0,21 \mathrm{x}-0,74)$ und fehlte bei den anderen Genotypen $(\mathrm{H}, 0,97$; 95\%-KI 0,62-1,54).

Schlussfolgerungen: Die frühe Gabe von Probiotika kann das Risiko für Inselautoimmunität bei Kindern mit hohem genetischem Risiko vermindern. Diese Ergebnisse müssen in weiteren Studien bestätigt werden, bevor die frühe Gabe von Probiotika empfohlen werden kann.

\section{- Kommentar von Prof. Dr. med. Nanette C. Schloot}

\section{Noch einige offene Fragen}

Hier gibt es wieder einen Lichtblick für eine vielleicht doch mögliche primäre Typ-1-Diabetesprävention. Allerdings bedarf diese Beobachtung einer Bestätigung, bevor Probiotika als supplementäre Gabe bei Kindern in den ersten 4 Lebenswochen empfohlen werden kann. Auch würde man natürlich gern den Mechanismus dahinter verstehen und wissen, wieviele Probiotika wit häufig empfohlen werden könnten: tägliche Gabe? Wöchentliche Gabe? Einmalige Gabe?

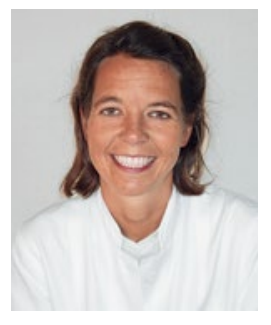

Prof. Dr. med. Nanette C. Schloot

Deutsches Diabetes-Zentrum

Institut für Klinische Diabetologie an der Heinrich-Heine Universität Düsseldorf Nanette.Schloot@DDZ.uni-duesseldorf.de 